\title{
Predictive molecular profiling in blood of healthy vasospastic individuals: clue to targeted prevention as personalised medicine to effective costs
}

\author{
Kristina Yeghiazaryan • Josef Flammer • \\ Olga Golubnitschaja
}

Received: 30 April 2010 /Accepted: 1 June 2010 / Published online: 29 June 2010

(C) European Association for Predictive, Preventive and Personalised Medicine 2010

\begin{abstract}
Paradigm change from late interventional approach to predictive diagnostics followed by targeted prevention before manifest pathology, presents innovative concept for advanced healthcare. Preselection of healthy but pathology-predisposed individuals is the primary task in the overall action. Vasospasm is a frequent syndrome defined as an inappropriate constriction or insufficient dilatation in microcirculation. Vasospastic individuals are considered as healthy subpopulation predisposed to several pathologies including neurodegeneration. Clinical observations, subcellular imaging and "gene hunting"-investigations provide evidence for vasospasm as predisposition to glaucoma; development of further related pathologies cannot be excluded. Predictive molecular-profiling in blood can specify individual predisposition for effective prevention.
\end{abstract}

K. Yeghiazaryan $\cdot$ O. Golubnitschaja $(\triangle)$

Department of Radiology, Rheinische Friedrich-Wilhelms-

University of Bonn,

Sigmund-Freud-Str. 25,

53105 Bonn, Germany

e-mail: Olga.Golubnitschaja@ukb.uni-bonn.de

K. Yeghiazaryan · J. Flammer $\cdot$ O. Golubnitschaja

European Association for Predictive,

Preventive \& Personalised Medicine

URL: www.epmanet.eu

\section{J. Flammer}

Department of Ophthalmology, University of Basel,

Basel, Switzerland
Keywords Predisposition in healthy individuals · Vascular deregulation - Predictive diagnostics · Blood biomarkers . Targeted prevention $\cdot$ Personalised medicine

Vasospastic Syndrome: definition, prevalence, detection

Vasospastic Syndrome or vascular deregulation (VD) is defined as an inappropriate constriction or insufficient dilatation in the microcirculation (see Fig. 1).

Generally, vasospasm is considered as primary and secondary one. Secondary VD is due to other diseases such as autoimmune one. Primary VD is prevalent in younger subpopulation, can potentially predispose to several disorders being, therefore, particularly attractive for predictive diagnostics and individualised treatment. Primary VD demonstrates following particularities:

- it occurs more frequent in females manifested in puberty and moderating with age

- this phenomenon is even more frequent in Japanese population compared to Caucasian [2]

- usually academics are more affected by VD [3]

- to clinical signs belong an inborn increased sensitivity to any kind of stress provocation (mechanical, cold, emotion, etc.), altered drug sensitivity, frequently cold extremities, altered sleep behaviour, reduced feeling of thirst, low blood-pressure, reduced body-mass-index, more frequent migraine compared to general population $[1,4,5]$

- Compared to general population, vasospastic individuals tend to a meticulous personality and successful professional career [6]. 


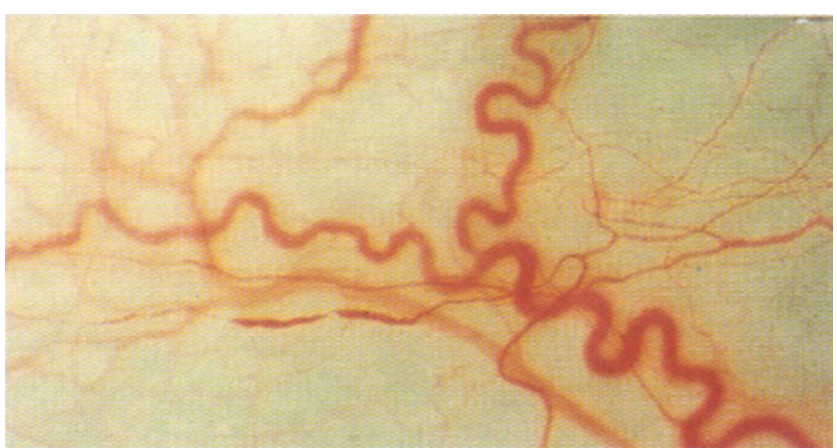

Fig. 1 Alterations in conjunctival vessels in patients with vasospasm [1]

A valuable diagnostic tool for the ascertainment of vasospastic diathesis is the nailfold capillary microscopy (see Fig. 2).

The best known blood-related risk factor is an increased plasma level of endothelin-1 [1]. Since Vasospastic Syndrome is a frequent phenomenon in young subpopulations, this makes the task of prediction and targeted prevention of "down-stream" related pathologies particularly attractive from several points of view including economical aspects.

\section{What is the impact of vascular deregulation in glaucoma pathology?}

A wealth of literature points to the importance of haemodynamics in glaucoma pathology. Vasospasm is frequently observed in glaucoma patients [1,7]. Ocular ischemia resulting from blood-flow deficits may play a major role in the initiation of glaucoma: hypoxia, followed by high secretion of excitatory amino acids and elevated levels of intracellular calcium results in the process of retinal ganglion cell death [8-10]. In our previous studies, we have demonstrated stable alterations in gene expression of circulating leucocytes isolated from glaucoma patients compared to healthy controls [11-14]. Further, significant similarities in expression profiles of circulating leucocytes between vasospastic individuals and glaucoma patients have been recently published [15]. However, the same publication reports also significant dissimilarities of molecular patterns as compared to both glaucoma patients and healthy controls; consequently, a development of both degenerative and non-degenerative pathologies different from glaucomatous optic nerve degeneration but related to primary vasospasm cannot be excluded in vasospastic individuals.

\section{What is the potential impact of prediction and prevention of glaucoma in healthy vasospastic individuals?}

Worldwide, 67 million patients are affected by the neurodegenerative eye disease glaucoma. Glaucomatous optic neuropathy (GON) is the second leading cause of permanent vision loss. GON is a chronic degenerative process, the onset of which is not possible to monitor by currently existing diagnostic tools. Early treatment has been reported to be highly beneficial for well-timed treatment measures to slow-down the disease progression [16]. As review in this journal-issue [17], molecular pathomechanisms of glaucoma demonstrate both a considerable overlap and remarkable particularities to some other neurodegenerative disorders such as Alzheimer's and Parkinson's diseases. Thus versus controls, the neuronal thread protein (NTP) demonstrates enhanced expression levels in glaucoma, patients with Down Syndrome, Alzheimer's and some other neurodegenerative diseases indicating the axonal lesions. However, whereas the accumulation of TAUprotein is characteristic for Alzheimer's disease and other tauopathies, glaucoma patients do not demonstrate an increase in the target protein versus controls [16, 18]. Therefore, a potential predisposition of vasospastic individ-
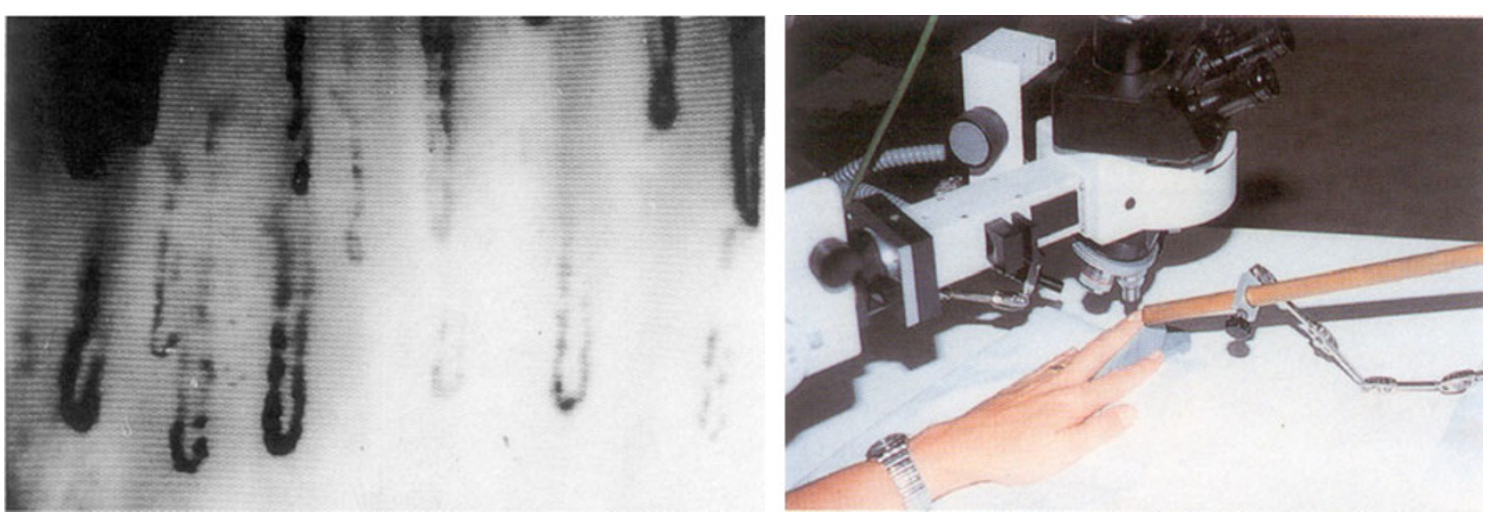

Fig. 2 Nailfold capillary microscopy (right) enables the monitoring of microcirculation and cold provocation of vasospasm in fingers (left) [1] 
uals to related pathologies should be thoroughly examined. In this context, molecular pathways involved in vasospastic deregulation should be investigated from viewpoint of

- identification of possible similarities as well as dissimilarities in molecular pathways between healthy vasospastic individuals and potential related pathologies developed later in life

- specificity for predictive diagnostics of glaucoma pathology in predisposed vasospastic individuals should be strictly validated against several control groups including other neuro/degenerative diseases

- selection of molecular targets should be performed for vasospastic individuals in favour of non-invasive (blood test) diagnostic approaches followed by personalised treatment towards individual predisposition to single pathologies.

A monitoring of the pathology-specific molecular patterns is particularly valuable to develop reliable diagnostic approaches before the manifest pathology. Predictive tests can specify individual predisposition for well-timed preventive measures.

\section{Similarities in subcellular images of DNA-damage and -repair capacity in vasospasm and glaucoma}

Research work focused on the ex vivo comparative investigations of DNA damage in circulated leucocytes (CL) isolated from patients with glaucoma demonstrated significantly enhanced DNA damage compared to both healthy vasospastic and non-vasospastic individuals [19]. Comparative "Comet Assay" analysis revealed patterns of comets typical for glaucoma patients as shown in Fig. 3.

Although DNA damage in the vasospastic nonglaucomatous group is not found to be significantly increased versus healthy controls, DNA from vasospastic individuals showed highly group-specific comet-patterns with the degree of damage intermediate between healthy controls and glaucoma patients. These findings indicate "comet assay" profiling of DNA-damage in CL as a potentially powerful tool for the non-invasive early/ predictive molecular diagnostics of glaucoma disease in vasospastic individuals [18]. Furthermore, unrepaired DNA-damage in vasospastic individuals can lead to several pathologies different from glaucomatous optic nerve degeneration. This predisposition should be thoroughly investigated and the specificity of "Comet Assay"patterns of vasospastic individuals should be validated comparing with patterns of other degenerative and nondegenerative pathologies. Thus, "Comet Assay"-analysis as a suitable tool for biomarkers has also been suggested for another neurodegenerative disorder-Alzheimer's disease [21]. "Comet Assay"-analysis reveals enhanced DNA damage in both high- and normal-tension glaucoma [19]. Whether the level of DNA-damage correlates with disease severity, or not remains currently unclear. Further studies should also evaluate, whether a significant increase in DNA damage of leucocytes of glaucoma patients is caused by either disease specific stress factors, such as local ischemic/reperfusion events, and/or decreased capacity of DNA-repair machinery. There is some evidence for both eventualities: simultaneous up-regulation of $p 53$ (stress regulated gene) and down-regulation of $X P G C$ (essential member of DNA-repair machinery) have been ex vivo demonstrated in CL of glaucoma patients [22] and represent potential molecular blood markers for the disease.

\section{Similarities in expression patterns detected in circulating leucocytes of vasospastic individuals and glaucoma patients}

\section{D-PAGE}

Protein-patterns in circulating leucocytes demonstrate clear similarities between vasospasm and normal-tension glaucoma versus controls. Moreover, protein-clusters can be considered for predictive imaging of healthy vasospastic individuals to glaucoma as shown in Fig. 4.

\section{Expression array}

The image of hybridised "AtlasTM Human Cardiovascular Array" revealed similarities as well as alterations in expression-patterns among normal-tension glaucoma (NTG), high-tension glaucoma (HTG), and VD groups versus controls are summarised in Table 1.

Compared to the control group the expression of 146, 68, and 60 genes were found to be altered in NTG, HTG, and VD groups respectively; the same 53 genes were differentially expressed in both NTG and HTG groups versus controls. Among 146 genes differentially expressed specifically in NTG group we monitored 48 and 53 genes which were similarly expressed either in VD or in HTG groups respectively. Among 68 genes differentially expressed specifically in HTG group we found 43 genes to be similarly expressed in VD group only. The highest difference-146 genes - was found to be between NTG and control groups. In contrast, the lowest difference-21 genes-was demonstrated to be between VD and the overlap of NTG/HTG.

34 genes demonstrated similar expressional alterations in NTG, HTG, and VD groups versus controls as given in Table 2. As the differentially expressed overlap VD/NTG/ 
Fig. 3 Ex vivo "Comet Assay"analysis of DNA damage in circulating leucocytes of glaucoma patients and nonglaucomatous vasospastic individuals versus healthy controls [19]. Figures A-D give examples of images typical for a. control group, b. group of vasospastic individuals, c. group of normal-tension glaucoma patients, and $\mathbf{d}$. group of hightension glaucoma patients. Comet-patterns typical for healthy controls (A) show that chromosomal DNA is localised mainly to heads of comets (intact DNA). In contrast, images $\mathrm{B}, \mathrm{C}$, and D demonstrate clearly damaged DNA (visible comettails and diffuse comet-heads). Evaluation of the shape of a comet allows for assessment of DNA damage. Individual migration patterns are demonstrated in groups corresponding to the images A-D by the evaluation of comet-classes (class number progression corresponds to increasing damage degree) as described earlier [20]. Figure E shows mean values calculated for relative DNA damage in following groups as compared to the control group (100\%):

VI-vasospastic individuals (102 $\pm 7 \%), N T G$ normal-tension glaucoma ( $160 \pm 13 \%)$, and $H T G$ high-tension glaucoma (128 \pm $10 \%)[18]$

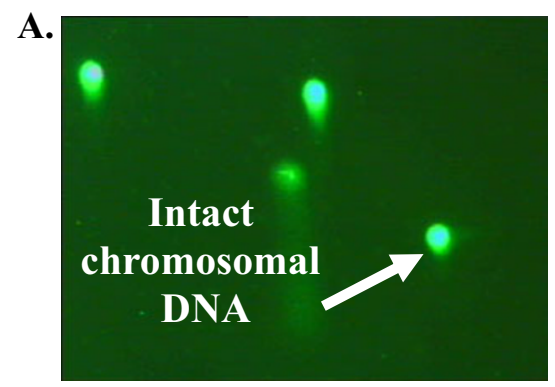

B.
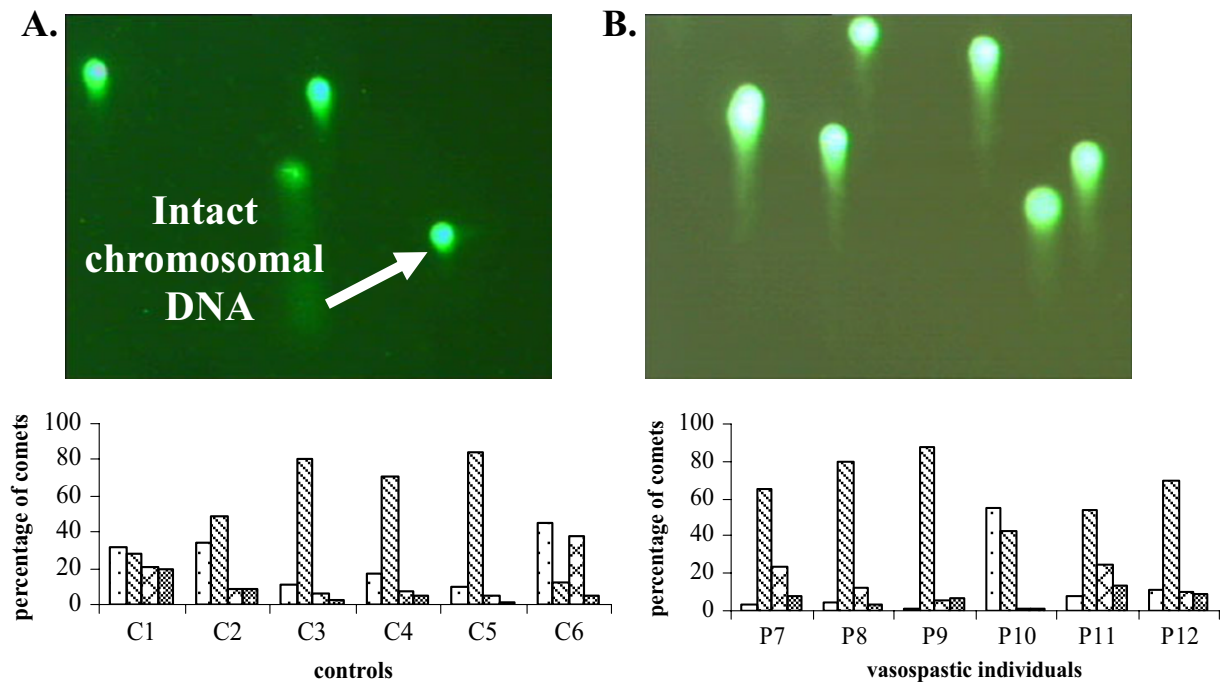

$\square$ class $1 \quad$ class $2 \square$ class $3 \quad$ class 4
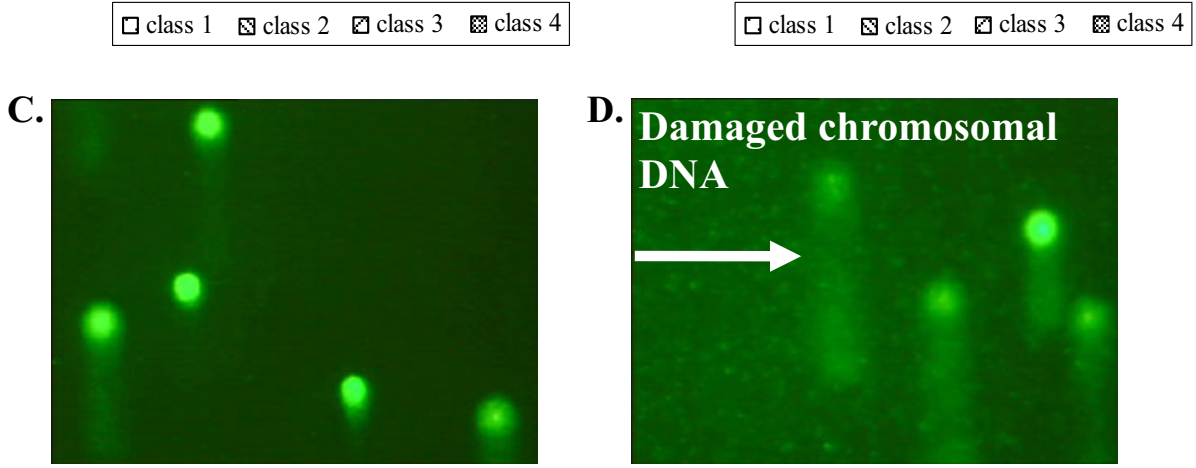

D. Damaged chromosomal
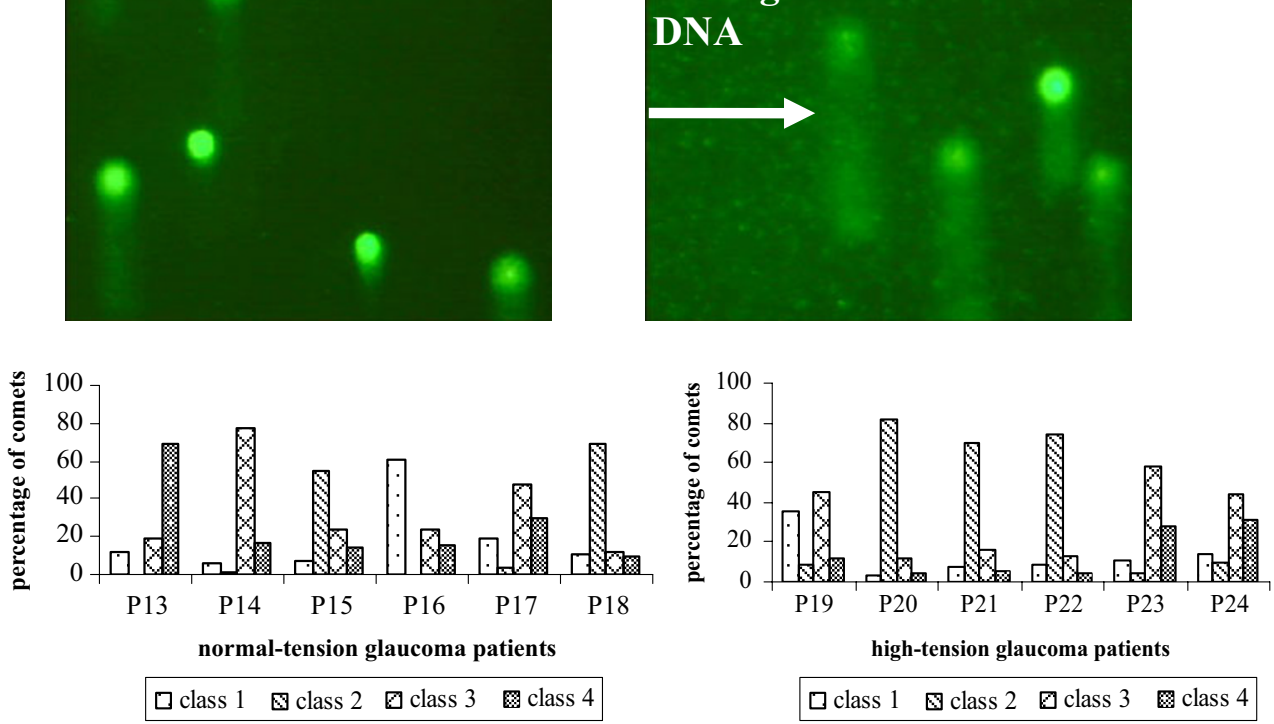

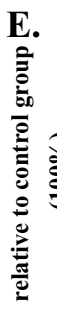

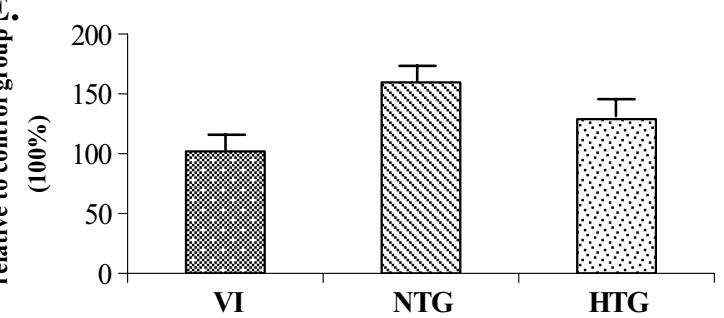

HTG was compared with the control group, following most significant difference was monitored:

- $\quad$ P2Y purinoreceptor 7

- $\mathrm{Na}^{+} / \mathrm{Ca}^{2+}$ exchange protein $1\left(\mathrm{Na}^{+} / \mathrm{Ca}^{2+} \mathrm{EP} 1\right)$

- Intercellular adhesion molecule 1 (ICAM1)

- The cluster of the tissue remodelling metalloproteinases.
This group of gene-transcripts is proposed to be the reliable target to design advanced diagnostic tools for predictive glaucoma diagnosis in healthy vasospastic individuals [15].

P2Y purinoreceptor is upregulated in vasospastic individuals and glaucoma patients The movement of leucocytes 


\section{A. Top}
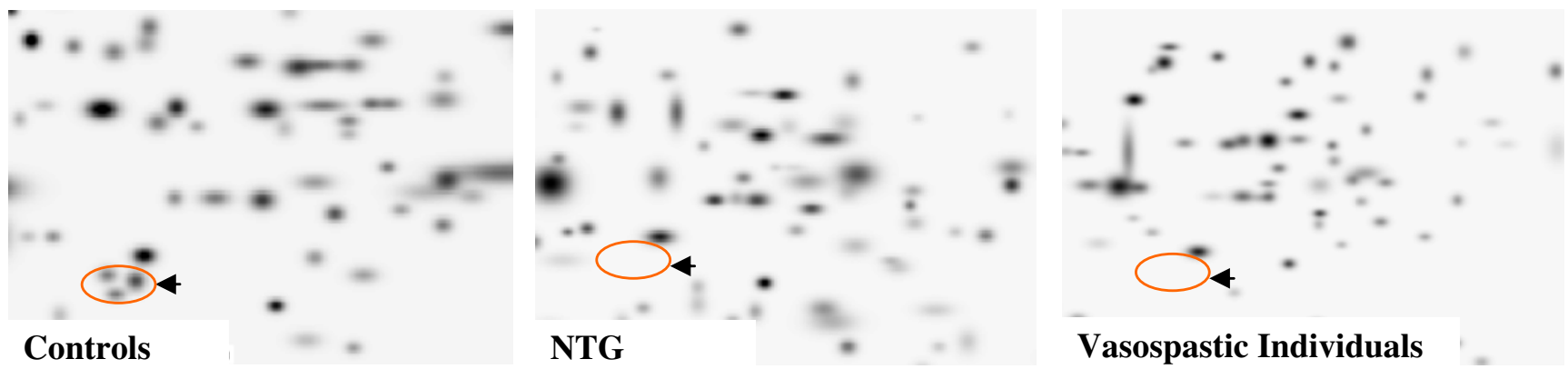

Vasospastic Individuals
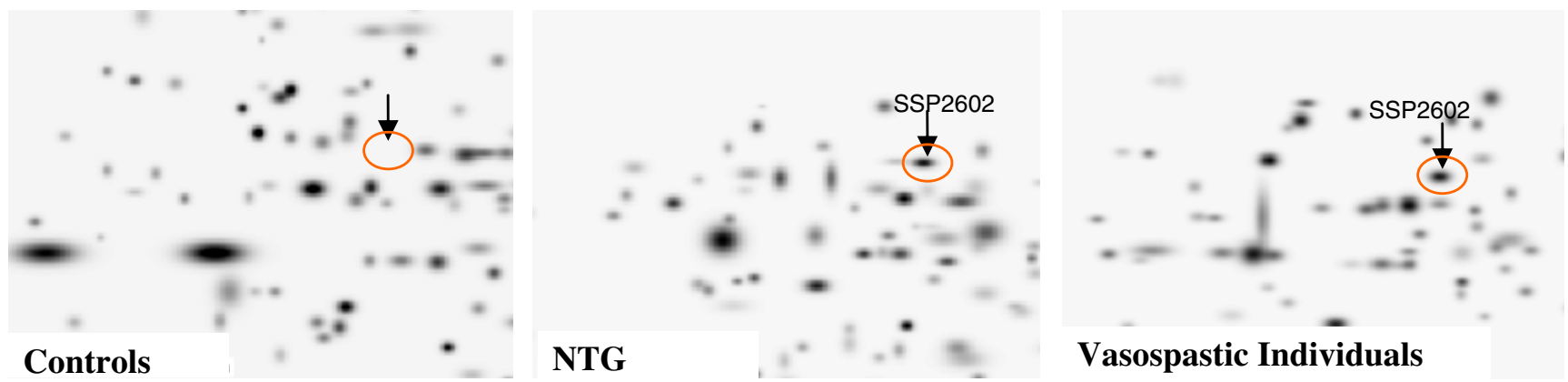

Vasospastic Individuals

\section{B. Bottom}

Fig. 4 Proteomics-imaging of blood-biomarkers (ex vivo identification in circulating leucocytes) specific for normal-tension glaucoma (NTG). A. Top: The pathology-specific protein-cluster is completely suppressed in both NTG and vasospasm in contrast to controls.

from blood into tissue is regulated by local production of chemo-attractants - diverse molecules, the chemotactic signal of which is transmitted by G-protein-coupled purinoceptor family P2Y. These receptors respond to chemotactic signals of traumatic, infectious, post-ischemic, autoimmune, and various toxic injuries. Extracellular nucleotides released from the activated platelets and other damaged cell types exacerbate the inflammatory response by cell specific leukotrene generation [23]. Thus, neutrophils generate leukotrienes B4 (LTB4) which are involved in the genesis of inflammation and oedema because of their effect on

Table 1 Numbers of genes an expression of which is either differential or equal among the groups tested as shown by "Expression array". Thereby, 108 genes were found to be differentially expressed between NTG and HTG groups. 34 genes demonstrated similar alteration for vasospastic individuals (VD) and both glaucoma-patient groups when compared to the healthy controls (see these genes listed in Table 2)

\begin{tabular}{lll}
\hline Differential to control & VD equal to & VD Differential to \\
\hline $\mathrm{NTG} \rightarrow 146$ & Control $\rightarrow 528$ & $\mathrm{NTG} \rightarrow 109$ \\
$\mathrm{HTG} \rightarrow 68$ & $\mathrm{NTG} \rightarrow 48$ & $\mathrm{~K} \rightarrow 60$ \\
$\mathrm{VD} \rightarrow 60$ & $\mathrm{HTG} \rightarrow 43$ & $\mathrm{HTG} \rightarrow 43$ \\
$\mathrm{NTG}=\mathrm{HTG} \rightarrow 53$ & $\mathrm{NTG}=$ HTG $\rightarrow$ 34 & $\mathrm{NTG}=\mathrm{HTG} \rightarrow 21$ \\
\hline
\end{tabular}

B. Bottom: The marked protein SSP2602 is highly up-regulated in both NTG and vasospasm; this protein normally is not expressed by circulating leucocytes of controls - healthy individuals without vasospasm [22]

vascular permeability, plasma extravasation, diapedesis of white blood cells, and their important role in adaptive immune responses as reviewed by Di Gennaro et al. [24]. Specifically, a highly enhanced concentration of leukotrienes B4 and C4 has been observed in CSF of patients with multiple sclerosis [25]. The member of leukotrienes receptors family - LTB4 receptor or P2Y purinoceptor $7-$ for the first time has been isolated from human erythroleukaemia cell cDNA library [26]. The stimulation of monocytes, neutrophils, and endothelial cells was suggested to be a physiological role for the LTB4 receptor [27]. There is a growing body of evidence indicating an important role of LTB4 receptors in regulation of pathologic inflammation. Particularly using animal inflammatory models a reduced disease severity has been shown as LTB4 receptor antagonists have been applied; the same effect has been observed in mice with target deletion of BLT1-a high-affinity LTB4 receptor primarily expressed in leucocytes [28]. Furthermore, some studies support a potential role of P2Y receptors in controlling intraocular pressure, although additional investigations of the issue are necessary [29].

ICAM-1 is upregulated in vasospastic individuals and glaucoma patients Neutrophil-endothelium interactions are implicated in pathological alterations of blood vessel 
Table 2 Differentially expressed genes (altogether 34 ones as also summarised in Table 1) versus controls, the transcriptional levels of which were similar for VD, NTG and HTG groups [18]

\begin{tabular}{|c|c|c|c|c|}
\hline $\begin{array}{l}\text { Double-spot position } \\
\text { in "EA"-image exp. } \\
\text { difference vs. control }\end{array}$ & $\begin{array}{l}\text { Name of gene as given } \\
\text { in "Atlas }{ }^{\mathrm{TM}} \text { Human } \\
\text { Cardiovascular Array" }\end{array}$ & $\begin{array}{l}\text { GenBank } \\
\text { Accession }\end{array}$ & $\begin{array}{l}\text { SwissProt } \\
\text { Accession }\end{array}$ & $\begin{array}{l}\text { Gene/Protein } \\
\text { Classification }\end{array}$ \\
\hline $\begin{array}{l}\text { A7d } \\
\text { increased }\end{array}$ & $\begin{array}{l}\text { P2Y purinoceptor } 7(\mathrm{P} 2 \mathrm{Y} 7) \text {; } \\
\text { leukotriene B4 receptor; Chemoattractant } \\
\text { receptor-like1 (CMKRL1) }\end{array}$ & U41070 & $\begin{array}{l}\text { Q15722 } \\
\text { Q13305 } \\
\text { Q92641 }\end{array}$ & $\begin{array}{l}\text { Other receptors (by } \\
\text { Ligands) } \\
\text { G Protein-Coupled Receptors }\end{array}$ \\
\hline $\begin{array}{l}\text { A7e } \\
\text { increased }\end{array}$ & $\begin{array}{l}\text { Retinoic acid (Vitamin-A1-Säure) } \\
\text { receptor gamma } 1 \text { (RAR-gamma } \\
\text { 1; RARG) }\end{array}$ & $\begin{array}{l}\text { M24857 } \\
\text { M38258 } \\
\text { M57707 } \\
\text { M32074 }\end{array}$ & P13631 & $\begin{array}{l}\text { Transcription Activator } \\
\quad \text { \& Repressors } \\
\text { Hormone Receptors } \\
\text { Nuclear Receptors }\end{array}$ \\
\hline $\begin{array}{l}\text { B1n } \\
\text { increased }\end{array}$ & $\begin{array}{l}\text { Androgen receptor coactivator } \\
70-\mathrm{kDa} \text { subunit (ARA70) }\end{array}$ & L49399 & Q13772 & $\begin{array}{l}\text { Transcription Activator } \\
\text { \& Repressors }\end{array}$ \\
\hline $\begin{array}{l}\text { B4c } \\
\text { increased }\end{array}$ & $\begin{array}{l}\text { G protein-activated inward potassium } \\
\text { channel } 4 \text { (GIRK4); heart K+/ATP } \\
\text { channel (KATP1); cardiac inward } \\
\text { rectifyer (CIR); KIR3.4 }\end{array}$ & U39195 & $\begin{array}{l}\text { P48544 } \\
\text { Q92807 }\end{array}$ & Voltage-gated Ion Channels \\
\hline $\begin{array}{l}\text { B4d } \\
\text { increased }\end{array}$ & $\begin{array}{l}\text { Sodium/calcium exchanger } 1 \text { precursor; } \\
\mathrm{Na}+/ \mathrm{Ca} 2+\text {-exchange protein } 1\end{array}$ & M91368 & P32418 & Symporters \& Antiporters \\
\hline $\begin{array}{l}\text { B4e } \\
\text { increased }\end{array}$ & $\begin{array}{l}\text { Multidrug resistance protein } 3 \text { (MDR3); } \\
\text { P-Glycoprotein } 3 \text { (PGY3) }\end{array}$ & M23234 & P21439 & $\begin{array}{l}\text { Drug-Resistance proteins } \\
\text { Xenobic Transporters } \\
\text { ABC transporters }\end{array}$ \\
\hline $\begin{array}{l}\text { B5f } \\
\text { increased }\end{array}$ & Endothelial nitric oxide synthase (EC-NOS) & M93718 & P29474 & $\begin{array}{l}\text { Other Metabolism Enzymes } \\
\text { Other Intracellular Transducers, } \\
\text { Effectors \& Modulators }\end{array}$ \\
\hline $\begin{array}{l}\text { B6d } \\
\text { increased }\end{array}$ & $\begin{array}{l}\text { Intercellular adhesion molecule } 1 \text { precursor } \\
\text { (ICAM1); major group rhinovirus receptor; } \\
\text { CD54 antigen }\end{array}$ & J03132 & P05362 & Matrix Adhesion Receptors \\
\hline $\begin{array}{l}\mathrm{B} 7 \mathrm{~g} \\
\text { increased }\end{array}$ & Calcium \& integrin-binding protein (CIB) & U85611 & Q99828 & Calcium-Binding proteins \\
\hline $\begin{array}{l}\mathrm{C} 1 \mathrm{~g} \\
\text { increased }\end{array}$ & Cadherin 7 (CDH7) & AF047826 & O60574 & $\begin{array}{l}\text { Cell Surface Antigens } \\
\text { Cell-Cell Adhesion } \\
\text { Receptors }\end{array}$ \\
\hline $\begin{array}{l}\text { C1h } \\
\text { increased }\end{array}$ & Intestinal peptide-associated transporter 1 (HPT1) & U07969 & Q12864 & $\begin{array}{l}\text { Other Cell Adhesion proteins } \\
\text { Other Cell Adhesion Proteins } \\
\text { Other Facilitated } \\
\text { Diffusion proteins }\end{array}$ \\
\hline $\begin{array}{l}\mathrm{C} 2 \mathrm{i} \\
\text { increased }\end{array}$ & GAP junction alpha- 5 protein & L34954 & P36382 & $\begin{array}{l}\text { Cell-Cell Adhesion Receptors } \\
\text { Other Membrane } \\
\text { Channels \& Transporters }\end{array}$ \\
\hline $\begin{array}{l}\mathrm{C} 3 \mathrm{~m} \\
\text { increased }\end{array}$ & $\begin{array}{l}\text { Integrin beta } 2 \text { (ITGB2); cell surface adhesion } \\
\text { glycoprotiens LFA-1/CR3/p150, } 95 \text { beta } \\
\text { subunit precursor; CD18 antigen; } \\
\text { Complement receptor C3 beta subunit }\end{array}$ & M15395 & $\begin{array}{l}\text { P05107 } \\
\text { Q16418 }\end{array}$ & Cell-Cell Adhesion Receptors \\
\hline $\begin{array}{l}\text { D1 m } \\
\text { increased }\end{array}$ & $\begin{array}{l}\text { Cardiac LIM domain protein; muscle LIM } \\
\text { protein; cystein-rich protein } 3 \text { (CRP3); } \\
\text { LIM-only protein } 4\end{array}$ & U49837 & P50461 & $\begin{array}{l}\text { Basic Transcription Factors } \\
\text { Other Transcription Proteins } \\
\text { DNA Synthesis, Recombination } \\
\text { \& Repair Proteins }\end{array}$ \\
\hline $\begin{array}{l}\text { D1n } \\
\text { increased }\end{array}$ & Cardiotrophin-1 (CT1) & U43030 & Q16619 & $\begin{array}{l}\text { Growth Factors, cytokines \& } \\
\text { Chemokines }\end{array}$ \\
\hline $\begin{array}{l}\mathrm{D} 2 \mathrm{n} \\
\text { increased }\end{array}$ & Matrix metalloproteinase 16 (MMP-16) & D83646 & P51512 & $\begin{array}{l}\text { Chromatin Proteins } \\
\text { Metalloproteinases }\end{array}$ \\
\hline
\end{tabular}


Table 2 (continued)

\begin{tabular}{|c|c|c|c|c|}
\hline $\begin{array}{l}\text { Double-spot position } \\
\text { in "EA"-image exp. } \\
\text { difference vs. control }\end{array}$ & $\begin{array}{l}\text { Name of gene as given } \\
\text { in "Atlas }{ }^{\mathrm{TM}} \text { Human } \\
\text { Cardiovascular Array" }\end{array}$ & $\begin{array}{l}\text { GenBank } \\
\text { Accession }\end{array}$ & $\begin{array}{l}\text { SwissProt } \\
\text { Accession }\end{array}$ & $\begin{array}{l}\text { Gene/Protein } \\
\text { Classification }\end{array}$ \\
\hline D4a & TIMP-3 & U14394 & P35625 & $\begin{array}{l}\text { Extracellular Matrix } \\
\text { Proteins }\end{array}$ \\
\hline increased & & & & Proteinase Inhibitor \\
\hline $\mathrm{D} 4 \mathrm{~b}$ & TIMP-4 & U76456 & Q99727 & $\begin{array}{l}\text { Extracellular Matrix } \\
\text { Proteins }\end{array}$ \\
\hline increased & & & & Proteinase Inhibitor \\
\hline $\begin{array}{l}\text { D4d } \\
\text { increased }\end{array}$ & $\begin{array}{l}\text { Sterol regulatory element-binding } \\
\text { transcription factor } 1\end{array}$ & U00968 & P36956 & $\begin{array}{l}\text { Basic trnascription Factors } \\
\text { Other Apoptosis- } \\
\text { Associated Proteins }\end{array}$ \\
\hline $\begin{array}{l}\mathrm{D} 4 \mathrm{e} \\
\text { increased }\end{array}$ & $\begin{array}{l}\text { Sterol regulatory element-binding } \\
\text { transcription factor } 2\end{array}$ & U02031 & Q12772 & $\begin{array}{l}\text { Basic trnascription Factors } \\
\text { Other Apoptosis- } \\
\text { Associated Proteins }\end{array}$ \\
\hline \multirow[t]{2}{*}{$\begin{array}{l}\text { D5 } \mathrm{h} \\
\text { increased }\end{array}$} & Rab geranylgeranyl transferase bety subunit & Y08201 & P53611 & $\begin{array}{l}\text { Trafficking \& Targeting } \\
\text { Proteins }\end{array}$ \\
\hline & & & Q92697 & $\begin{array}{l}\text { Protein Modification } \\
\text { Enzymes } \\
\text { GTP/GDP Exchangers } \\
\text { \& GTPase Activity } \\
\text { Modulators }\end{array}$ \\
\hline \multirow[t]{3}{*}{$\begin{array}{l}\text { D6 } \mathrm{m} \\
\text { decreased }\end{array}$} & $\begin{array}{l}\text { Muscle-specific DNase I-like precursor } \\
\text { (DNase } 1 \text { L1; DNL } 1 \text { L); Dnase X }\end{array}$ & X90392 & P49184 & $\begin{array}{l}\text { DNA Synthesis, } \\
\text { Recombination \& } \\
\text { Repair Proteins }\end{array}$ \\
\hline & & L40817 & & $\begin{array}{l}\text { Apoptosis-Associated } \\
\text { Proteins }\end{array}$ \\
\hline & & U06846 & & \\
\hline $\begin{array}{l}\text { E1b } \\
\text { increased }\end{array}$ & $\begin{array}{l}\text { Lanosterol synthase (LSS); oxidosqualene } \\
\text { lanosterol cyclase (OSC) }\end{array}$ & D63807 & P48449 & $\begin{array}{l}\text { Complex Lipid } \\
\text { Metabolism }\end{array}$ \\
\hline $\begin{array}{l}\text { E3n } \\
\text { increased }\end{array}$ & NADPH-cytochrome p450 reductase & S90469 & $\begin{array}{l}\text { Q16455 } \\
\text { P16435 }\end{array}$ & Xenobic Metabolism \\
\hline $\begin{array}{l}\text { E4 g } \\
\text { increased }\end{array}$ & $\begin{array}{l}\text { Steroid } 5 \text { alpha reductase } 1 \text { (SRD5A1); } \\
\text { 3-oxo-5-alpha steroid } 4 \text { dehydrogenase } 1\end{array}$ & $\begin{array}{l}\text { M32313 } \\
\text { M68886 }\end{array}$ & P18405 & $\begin{array}{l}\text { Complex Lipid } \\
\text { Metabolism }\end{array}$ \\
\hline $\begin{array}{l}\mathrm{E} 4 \mathrm{~h} \\
\text { increased }\end{array}$ & $\begin{array}{l}\text { Steroid 5-alpha reductase } 2 \text { (SRD5A2); } \\
\text { 3-oxo-5-alpha steroid } 4 \text { dehydrogenase } 2\end{array}$ & M74047 & P31213 & $\begin{array}{l}\text { Complex Lipid } \\
\text { Metabolism }\end{array}$ \\
\hline $\begin{array}{l}\text { F2d } \\
\text { increased }\end{array}$ & Pregnane $\mathrm{X}$ receptor $(\mathrm{PXR})$ & AF061056 & O75469 & $\begin{array}{l}\text { Hormone receptors } \\
\text { Nuclear Receptors }\end{array}$ \\
\hline $\begin{array}{l}\mathrm{F} 2 \mathrm{e} \\
\text { increased }\end{array}$ & Estrogen-related receptor gamma & AF058291 & O75454 & $\begin{array}{l}\text { Hormone Receptors } \\
\text { Nuclear Receptors }\end{array}$ \\
\hline $\begin{array}{l}\mathrm{F} 2 \mathrm{f} \\
\text { increased }\end{array}$ & $\begin{array}{l}\text { Nuclear receptor subfamily } 4 \text { group A } \\
\text { member } 2 \text { (NR4A2); nuclear receptor-related } \\
\text { protein } 1 \text { (NURR1); transcriptionally inducuble } \\
\text { nuclear receptor (TINUR); NOT }\end{array}$ & X75918 & P43354 & $\begin{array}{l}\text { Hormone Receptors } \\
\text { Nuclear Receptors } \\
\text { Transcription Activators } \\
\text { \& Repressors }\end{array}$ \\
\hline $\begin{array}{l}\mathrm{F} 2 \mathrm{i} \\
\text { increased }\end{array}$ & $\begin{array}{l}\text { Orphan nuclear receptor TR4; nuclear receptor } \\
\text { subfamily } 2 \text { group c member } 2 \text { (NR2C2); TAK1 }\end{array}$ & U10990 & $\begin{array}{l}\text { P55092 } \\
\text { P49116 }\end{array}$ & $\begin{array}{l}\text { Orphan Receptors } \\
\text { Nuclear Receptors } \\
\text { Transcription Activators } \\
\text { \& Repressors }\end{array}$ \\
\hline $\begin{array}{l}\text { F3a } \\
\text { increased }\end{array}$ & RAR-related orphan receptor C & U16997 & P51449 & $\begin{array}{l}\text { Orphan Receptors } \\
\text { Nuclear Receptors } \\
\text { Transcription Activators } \\
\text { \& Repressors }\end{array}$ \\
\hline $\mathrm{F} 3 \mathrm{e}$ & LX receptor alpha (LXR alpha) & U22662 & Q13133 & Orphan Receptors \\
\hline
\end{tabular}


Table 2 (continued)

\begin{tabular}{|c|c|c|c|c|}
\hline $\begin{array}{l}\text { Double-spot position } \\
\text { in "EA"-image exp. } \\
\text { difference vs. control }\end{array}$ & $\begin{array}{l}\text { Name of gene as given } \\
\text { in "Atlas }{ }^{\mathrm{TM}} \text { Human } \\
\text { Cardiovascular Array" }\end{array}$ & $\begin{array}{l}\text { GenBank } \\
\text { Accession }\end{array}$ & $\begin{array}{l}\text { SwissProt } \\
\text { Accession }\end{array}$ & $\begin{array}{l}\text { Gene/Protein } \\
\text { Classification }\end{array}$ \\
\hline $\begin{array}{l}\text { F3i } \\
\text { increased }\end{array}$ & $\begin{array}{l}\text { Platelet-activating factor acetylhydrolase } \\
\text { IB alpha subunit }\end{array}$ & L13387 & P43034 & $\begin{array}{l}\text { Other Metabolism } \\
\text { Enzymes }\end{array}$ \\
\hline \multirow[t]{2}{*}{$\begin{array}{l}\text { F7c } \\
\text { increased }\end{array}$} & \multirow[t]{2}{*}{$\begin{array}{l}\text { Myocyte-specific enhancer factor } 2 \mathrm{~A}(\mathrm{MEF} 2 \mathrm{~A}) \text {; } \\
\text { serum response factor-like protein } 1\end{array}$} & \multirow[t]{2}{*}{ X68505 } & $\begin{array}{l}\text { Q02078 } \\
\text { Q14223 }\end{array}$ & \multirow[t]{2}{*}{$\begin{array}{l}\text { Basic Transcription } \\
\text { Factors }\end{array}$} \\
\hline & & & Q14224 & \\
\hline
\end{tabular}

function, potentially leading to circulatory disturbances [30]. Interactions between blood cells and the vessel wall result in endothelial dysfunction and injury leading to increased blood-brain barrier permeability and even oedema formation [31]. Penetration of leucocyte into inflamed areas involves complex interaction of leucocytes with endothelium through regulated expression of surface adhesion molecules. Found in this work to be highly expressed in VD, NTG and HTG groups ICAM-1 is believed to be largely responsible for the adhesion and trans-endothelial migration of leucocytes [32]. This is well in agreement with earlier developed strategies aimed at inhibition of endothelial interactions with leucocytes via use of adhesion molecule monoclonal antibodies, which successfully reduce cerebral ischemia/reperfusion injury, infarct size, and demonstrate a neuroprotective effect generally [33-35]. In our study, highly expressed ICAM-1 was found in leucocytes of glaucoma patients; in contrast, if any only traces of the target expression was detected in the leucocytes of healthy controls.

Sodium calcium exchanger (NCE) Many studies examined the levels of cytosolic $\mathrm{Ca}^{2+}\left(\left[\mathrm{Ca}^{2+}\right]_{\mathrm{c}}\right)$ and $\mathrm{Na}^{+}\left(\left[\mathrm{Na}^{+}\right]_{\mathrm{c}}\right)$ in human blood cells, whereby leucocytes have been the main target of studying the relationship between blood pressure and intracellular content of both ions as reviewed by Horiguchi et al. [36]. As it has been shown by Horiguchi et al., the resting $\left[\mathrm{Ca}^{2+}\right]_{\mathrm{c}}$ correlates well with $\mathrm{NCE}$ expression indicating NCE expression regulation to be an adaptive mechanism for $\mathrm{Ca}^{2+}$ extrusion mediation. The same study observed also a gender effect on $\left[\mathrm{Ca}^{2+}\right]_{\mathrm{c}} /$ $\left[\mathrm{Na}^{+}\right]_{\mathrm{c}}$ regulation in circulating leucocytes being in relationship with blood pressure. Further, the role of endothelial intracellular $\mathrm{Ca}^{2+}$ concentration in molecular mechanisms of vasoconstriction/vasodilatation has been intensively studied, and the functional association between P2Y purinoceptors, endothelial NO synthesis and calcium transport in terms of vascular regulation is well documented in the literature [37, 38]. Our findings here clearly demonstrate the up-regulation of both $\mathrm{P} 2 \mathrm{Y}$ purinoceptor and $\mathrm{Na}^{+} / \mathrm{Ca}^{2+}$ exchanger in circulating leucocytes of glaucoma patients as well as vasospastic individuals versus healthy controls.

Tissue remodelling metalloproteinases Significantly increased protein expression rates of both latent and active forms of metalloproteinases MMP-9 and MT1-MMP in circulating leucocytes correlate well with the enhanced levels of transcription and with glaucoma diagnosis [12]. Once activated, both hydrolases necessarily contribute to remodelling or even degeneration of the tissue whereto they are secreted by circulating leucocytes. This upregulation might be a consequence of repeated mild ischemia/reperfusion postulated for both vasospastic individuals and glaucoma patients [18]. However, the question as to whether or not there is a correlation between an increased MMPs activity and glaucoma severity should be further clarified.

Furthermore, the increased synthesis of tissue-remodelling hydrolases detected in blood of healthy vasospastic individuals can potentially lead to development of some other pathologies which have not been considered as related to vasospasm till now. To the potential list of them belong altered wound-healing, some types of organ-degeneration, increased metastases activity. Large-scale studies are essential to be preformed, in order to prove a potential impact of vasospasm for the above listed pathophysiologic processes / manifested pathologies. This allows a targeted prevention at the stage of pre-lesions, "upstream" disease manifestation.

\section{Concluding remarks}

- Expression similarities between glaucoma and VD versus controls indicate, on one side, a predisposition of VD individuals to glaucomatous damage, and, on the other side, an important role of vascular component in glaucoma pathology.

- Expression dissimilarities between VD and glaucoma patients might indicate some glaucoma-specific patho- 
mechanisms which are not involved in the stage of predisposition by VD.

- Both expression similarities and dissimilarities could be useful to ascertain predictive diagnosis of glaucoma.

- Molecular rearrangement in leucocytes of both VD and glaucoma patients has been shown to be typical for circulating leucocytes during vascular injury as reviewed by Kunapoli and Daniel [39] and includes

$\triangleright$ an up-regulated adhesive protein expression via ICAM1

$\triangleright$ an induced chemo-taxis via P2Y purinoceptors

$\triangleright$ a mobilisation of intracellular $\mathrm{Ca}^{2+}$ response via $\mathrm{Na}^{+} / \mathrm{Ca}^{2+}$ exchanger, and

$\triangleright$ the core of tissue remodelling metalloproteinases.

- Pathology-specific molecular and subcellular patterns may create the basis for the development of more specific and non-invasive molecular imaging technologies in early/predictive glaucoma diagnostics.

- Development of some other "down-stream" pathologies different from glaucoma but related to primary vasospastic syndrome cannot be excluded.

- Predictive molecular-profiling in blood can specify individual predisposition for effective prevention to lower costs compared to those for treatment of manifest pathologies.

\section{References}

1. Flammer J, Pache M, Resink T. Vasospasm, its role in the pathogenesis of diseases with particular reference to the eye. Prog Retin Eye Res. 2001;20:319-49.

2. Beltrame JF, Sasayama S, Maseri A. Racial heterogeneity in coronary artery vasomotor reactivity: differences between Japanese and Caucasian patients. J Am Coll Cardiol. 1999;33:1442-52.

3. Flammer J. Glaucoma. A guide for patients. An introduction for care providers. A quick reference. 3rd revised edition. Bern, Hogrefe \& Huber Publishers, 2006

4. Teuchner B, Orgül S, Ulmer H, et al. Reduced thirst in patients with a vasospastic syndrome. Acta Ophthalmol Scand. 2004;82:738-40.

5. Wunderlich K, Zimmerman C, Gutmann H, et al. Vasospastic persons exhibit differential expression of $\mathrm{ABC}$-transport proteins. Mol Vis. 2003;9:756-61.

6. Flammer J. Innovative glaucoma therapy. Ophthalmologe. 2001;98:923-4.

7. Finsterer J. Mitochondriopathies. Eur J Neurol. 2004;11:163-86.

8. Flammer J, Guthauser U, Mahler M. Do ocular vasospasms help cause low-tension glaucoma? Doc Ophthalmol Proc Ser. 1987;49:397-9.

9. Guthauser U, Flammer J, Mahler F. The relationship between digital and ocular vasospasm. Graefes Arch Clin Exp Ophthalmol. 1988;226:224-6.

10. Flammer J, Orgül S, Costa VP, et al. The impact of ocular blood flow in glaucoma. Prog Retin Eye Res. 2002;21:359-93.
11. Golubnitschaja O, Wunderlich K, Decker C, et al. Molecular imaging of perfusion disturbances in glaucoma. Amino Acids. 2002;23:293-9.

12. Golubnitschaja $\mathrm{O}$, Yeghiazaryan $\mathrm{K}$, Liu $\mathrm{R}$, et al. Increased expression of matrix metalloproteinases in mononuclear blood cells of normal-tension glaucoma patients. J Glaucoma. 2004;13:66-72.

13. Golubnitschaja-Labudova O, Liu R, Decker C, et al. Altered gene expression in lymphocytes of patients with normal-tension glaucoma. Curr Eye Res. 2000;21:867-76.

14. Wunderlich K, Golubnitschaja O, Pache $\mathrm{M}$, et al. Increased plasma levels of $20 \mathrm{~S}$ proteasome alpha-subunit in glaucoma patients: an observational pilot study. Mol Vis. 2002;8:431-5.

15. Yeghiazaryan K, Flammer J, Orgül S, et al. Vasospastic individuals demonstrate significant similarity to glaucoma patients as revealed by gene expression profiling in circulating leukocytes. Mol Vis. 2009;15:2339-48.

16. Golubnitschaja O, Flammer J. What are the biomarkers for Glaucoma? Surv Ophthalmol. 2007;52:S155-61.

17. Golubnitschaja O, Yeghiazaryan K, Flammer J. Key molecular pathways affected by glaucoma pathology: Is predictive diagnosis possible? The EPMA Journal. 2010; doi:10.1007/s13167-0100031-4.

18. Golubnitschaja O, Yeghiazaryan K, Orgül S, et al. What are the biomarkers for Glaucoma? In: Golubnitshaja O, editor. Predictive diagnostics and personalized treatment: dream or reality. New York: Nova Science Publishers; 2009. p. 375-96.

19. Moenkemann H, Flammer J, Wunderlich K, et al. Increased DNA breaks and up-regulation of both $\mathrm{G}(1)$ and $\mathrm{G}(2)$ checkpoint genes p21(WAF1/CIP1) and 14-3-3 sigma in circulating leukocytes of glaucoma patients and vasospastic individuals. Amino Acids. 2005;28:199-205.

20. Golubnitschaja O, Moenkemann H, Kim K, et al. DNA damage and expression of checkpoint genes p21(WAF1/CIP1) and 14-3-3 sigma in taurine-deficient cardiomyocytes. Biochem Pharmacol. 2003;66:511-7.

21. Migliore L, Fontana I, Colognato R, et al. Searching for the role and the most suitable biomarkers of oxidative stress in Alzheimer's disease and in other neurodegenerative diseases. Neurobiol Aging. 2005;26:587-95.

22. Golubnitschaja O, Yeghiazaryan K, Wunderlich K, et al. Disease proteomics reveals altered basic gene expression regulation in leukocytes of glaucoma patients. Proteomics Clin Appl. 2007;1:1316-23.

23. Kannan S. Amplification of extracellular nucleotide-induced leukocyte(s) degranulation by contingent autocrine and paracrine mode of leukotriene-mediated chemokine receptor activation. Med Hypotheses. 2002;59:261-5.

24. Di Gennaro A, Carnini C, Buccellati C, et al. Cysteinylleukotrienes receptor activation in brain inflammatory reactions and cerebral edema formation: a role for transcellular biosynthesis of cysteinyl-leukotrienes. FASEB J. 2004;18:842-4.

25. Rosnowska M, Cendrowski W, Sobczyk W. Leukotrienes B4 and $\mathrm{C} 4$ in cerebrospinal of patients with multiple sclerosis. Pol Merkur Lekarski. 1997;2:254-5.

26. Akbar GK, Dasari VR, Webb TE, et al. Molecular cloning of a novel P2 purinoceptor from human erythroleukemia cells. J Biol Chem. 1996;271:18363-7.

27. Dasari VR, Jin J, Kunapuli SP. Distribution of leukotriene B4 receptors in human hematopoietic cells. Immunopharmacology. 2000;48:157-63.

28. Tager AM, Luster AD. BLT1 and BLT2: the leukotriene B(4) receptors. Prostaglandins Leukot Essent Fatty Acids. 2003;69:123-34.

29. Pintor J, Peral A, Peláez T, et al. Presence of diadenosine polyphosphates in the aqueous humor: their effect on intraocular pressure. J Pharmacol Exp Ther. 2003;304:342-8. 
30. Akopov SE, Sercombe R, Seylaz J. Actions of plateletactivating factor on isolated rabbit basilar artery: modulation by activated polymorphonuclear leukocytes. J Vasc Res. 1995;32:49-57.

31. Akopov S, Sercombe R, Seylaz J. Cerebrovascular reactivity: role of endothelium/platelet/leukocyte interactions. Cerebrovasc Brain Metab Rev. 1996;8:11-94.

32. Butcher EC. Leukocyte-endothelial cell recognition: three (or more) steps to specificity and diversity. Cell. 1991;67:1033-6.

33. Barone FC, Feuerstein GZ. Inflammatory mediators and stroke: new opportunities for novel therapeutics. J Cereb Blood Flow Metab. 1999;19:819-34.

34. Chen H, Chopp M, Zhang RL, et al. Anti-CD11b monoclonal antibody reduces ischemic cell damage after transient focal cerebral ischemia in rat. Ann Neurol. 1994;35:458-63.
35. Connolly Jr ES, Winfree CJ, Springer TA, et al. Cerebral protection in homozygous null ICAM-1 mice after middle cerebral artery occlusion. Role of neutrophil adhesion in the pathogenesis of stroke. J Clin Invest. 1996;97:209-16.

36. Horiguchi M, Kimura M, Skurnick J, et al. Parameters of lymphocyte $\mathrm{Na}^{+}-\mathrm{Ca}^{2+}$ regulation and blood pressure: the gender effect. Hypertension. 1998;32:869-74.

37. Marrelli SP. Mechanisms of endothelial P2Y(1)- and P2Y(2)mediated vasodilatation involve differential $[\mathrm{Ca} 2+] \mathrm{I}$ responses. Am J Physiol Heart Circ Physiol. 2001;281:H1759-66.

38. Moncada S, Palmer RM, Higgs EA. Nitric oxide: physiology, pathophysiology, and pharmacology. Pharmacol Rev. 1991;43:109-42.

39. Kunapuli SP, Daniel JL. P2 receptor subtypes in the cardiovascular system. Biochem J. 1998;336:513-23. 